\title{
Germline mutations and their clinical applications in cancer
}

\author{
Jean-Sébastien Milanese ${ }^{1}$ iD \& Edwin Wang*,2,3 \\ ${ }^{1}$ Human Health Therapeutics, National Research Council Canada, 6100 Royalmount Ave, Montreal, H4P 2R2, Canada \\ ${ }^{2}$ Department of Biochemistry \& Molecular Biology, Medical Genetics, \& Oncology, University of Calgary, 3330 Hospital Dr NW, \\ Calgary, T2N 4N1, Canada \\ ${ }^{3}$ Alberta Children's Hospital Research Institute \& Arnie Charbonneau Cancer Research Institute, University of Calgary, 3330 \\ Hospital Dr NW, Calgary, T2N 4N1, Canada \\ *Author for correspondence: Tel.: +1 403220 6609; Fax: +1 4039891 9879; edwin.wang@ucalgary.ca
}

\section{"germline mutations not only increase cancer risk susceptibility but also act as modulators when shaping tumor evolution"}

First draft submitted: 21 February 2019; Accepted for publication: 22 February 2019; Published online: 20 March 2019

Keywords: cancer $\bullet$ genomics $\bullet$ germline mutations $\bullet$ system biology

Over the past few decades, researchers have mainly focused on cancer somatic mutations, while germline mutations have been largely ignored. Cancer is a stochastic event that arises from acquiring multiple somatic mutations; therefore, the scientific rationale for targeting and characterizing these mutations is perfectly founded. However, while we do agree with this concept in a general sense, we believe it is partially incomplete. One of the major risk factors in cancer is family history (or hereditary germline mutations). Germline mutations inherited from previous generations act as predispositions in individuals, increasing their susceptibility to develop cancer. A multitude of studies have already linked various germline predispositions in many genes (BRCA1, BRCA2, PTEN, TP53, KRAS, $A P C$, etc.), suggesting that germline mutations also impact tumor evolution [1-5]. Yet, still, many public databases, such as ICGC, have very little information concerning germline mutations ( 8857 germline profiles compared to 62329 somatic). Several years ago, we had proposed that pre-existing genetic variants could play important roles in shaping, or selecting, somatic mutations so that the selected mutated genes could work together with the pre-existing genetic variants to drive a transformation of a normal cell into a cancer cell [6]. Evidence from our most recent studies showed that inherited germline mutations not only increase cancer risk susceptibility but also act as modulators when shaping tumor evolution. We have shown that inheritably functional variants of breast cancer patients significantly predicted tumor recurrence [7,8], and the risk of developing breast, brain and other cancers [9]. Furthermore, we have shown that inheritably functional variants in natural killer cells (i.e., natural killer [NK] cells, an immune cell type) in cancer patients affected tumor-infiltrating lymphocytes (TILs) and survival [10]. These results bring a paradigm-shifting view about the roles of germline genomes in cancer. In the past decades, only a small fraction of cancer cases have been explained by germline dispositions; for example, a germline mutation in $B R C A 1 / 2$ is found in only $5-10 \%$ of breast and ovarian cancer patients. As the penetrance of other germline genetic defects is very small, their role in cancer development and metastasis have been hard to study and eventually ignored. Taking a systems biology approach, we showed that germline genomes play a much more important role in cancer development and metastasis than what might have been estimated by the cancer community.

\section{Germline mutations \& clinical outcome}

As mentioned above, until very recently, scientists have mainly focused on somatic mutations in cancer. Massive efforts, such as The Catalogue of Somatic Mutations in Cancer (COSMIC) [11] and TCGA, have been made to fully characterize the somatic mutational landscape across all cancer types. As a result, many mutational signatures to identify several cancer types, molecular subtypes and cancer prognosis have been discovered and have helped in cancer treatment and in clinical use [12-15]. Our initial hypothesis was that germline mutations played a much larger

Future $\because$ Medicine 
role in tumorigenesis than simply acting as predispositions. We reasoned that the collective impact of germline mutations, or more specifically the germline genomic landscape of a patient, could directly infer on the somatic mutational landscape. Although there is an event of randomness for a specific somatic mutation to be acquired by a cell, fitness of a somatic mutation will automatically be dependent and selected based on its pre-existing germline context. In simpler words, cancer cells utilize their host's historical and genetic background to maximize their survival. Therefore, the germline genomic landscape of a patient could inform us on tumor evolution, heterogeneity and even clinical outcome (such as recurrence or treatment response). To test this hypothesis, we compared the germline profiles of $755 \mathrm{ER}+$ breast cancer patients with their according recurrence status. We developed a machine-learning based algorithm, eTumorMetastasis, which utilizes the functional germline mutations to identify recurrence biomarkers $[7,8]$. Briefly, the model uses a network-based approach $[16,17]$ to transform functionally genetic variant's information on an ER+ breast cancer specific signaling network, from which we can identify biomarkers (or gene signatures) using machine learning [18]. The underlying hypothesis is that cancer cells are represented by a few cancer hallmarks, each of them composed of a few molecular/signaling networks. We proposed that 'network operational signatures' represent gene regulatory logics/strengths which enable us to quantify state transitions and measures hallmark traits. Thus, sets of genomic alterations associated with network operational signatures could be linked to the state/measure of hallmark traits. The network operational signature transforms genotypic data (i.e., genomic alterations) to regulatory phenotypic profiles (i.e., regulatory logics/strengths) and then to cellular phenotypic profiles (i.e., hallmark traits) which lead to clinical phenotypic profiles (i.e., a collection of hallmark traits). More details about these concepts can be found in Wang et al. [6]. The biomarkers here are the network operational signatures. Strikingly, we were able to predict low-risk samples with an accuracy as high as 94\%. Considering germline mutations can be obtained from sequencing a blood or saliva sample, this provides a new avenue in a clinical environment where germline mutations could be used to classify patients at diagnosis in a noninvasive manner, allowing physicians not to overtreat patients.

\section{Germline implications in the immune system}

Following the recurrence risk prediction results, we thoroughly analyzed our gene signatures. Enrichment analysis revealed interesting correlations between signature genes and immune systems (mostly antigen processing, $\mathrm{T}$ cell stimulation and NK cells) [8]. Hence, we hypothesized that germline mutations could impair leukocyte genes before tumorigenesis which would lead to a weakened immune response. Based on recurrence predictions, we compared leukocyte metagene expression as well as leukocyte cell fractions between both predicted groups. These results highlighted that germline mutations already predispose the immune system of patients, leading to a weaker immune response which, in turn, promotes a more favorable micro-environment for tumorigenesis and increases patient's relapse risk.

Another aspect of our study focused on classifying tumor immune microenvironments (TIMEs). Interestingly, we were able to classify TIMEs into three distinct groups commonly found in all cancer types: TIME-rich (or 'immunehot' tumors), TIME-intermediate (or 'immune-cold' tumors) and TIME-poor (or 'immune-desert tumors') [10]. By comparing germline genomics of the three TIME groups, we found that the number of inherited defected NK-cell related genes was negatively correlated with patient survival, TIL abundance in TIMEs, suggesting that inherited defects in NK cells alone were sufficient to shape TILs recruitment and therefore, clinical outcome and immunotherapy response. These results have major implications in the identification of high-risk individuals based on germline genomes and in current immunotherapy approaches. Adoptive transfer of healthy NK cells combined with anti-PD-1 or chimeric antigen receptor (CAR) T cell therapies would allow a conversion of TIME-intermediate and TIME-poor tumors into TIME-rich tumors, improving patient survival.

\section{Looking forward}

High throughput sequencing has created a shift in traditional medicine. Whole exome sequencing is now very affordable, which is leading to an enormous amount of data available. As a result, massive cohorts from hospitals are now being sequenced extensively [19]. However, one of major issues in personalized medicine is the ability to use this genomic information and translate it into predictive models or genomic tests that can be used into clinics on a daily basis. In our previous work, we have demonstrated that germline mutations have a strong correlation with patient outcome, that the germline genetic landscape modulates tumorigenesis and recurrence and that this landscape is enriched in immune system-related genes directly affecting TIMEs. Altogether, our results suggest that germline genomes, biomarkers or even TIME profile could be used in either cancer prognosis or diagnosis. 
Unlike gene expression, germline genetic information can be obtained from a simple and noninvasive blood or saliva sample. In current breast cancer protocols, if patients meet the criteria, physicians use OncotypeDx [20,21] to measure the risk of recurrence and/or if patients will benefit from adjuvant chemotherapy treatment which leads to an optimal adjustment per individual. As of now, germline genetic testing is scarcely used and is usually focused on a small panel of genes. We envision that whole exome germline genetic testing could be used in a similar fashion to OncotypeDx, which would provide improvements for personalized medicine in areas such as drug response, overtreatment and, from a financial point of view, healthcare associated costs. Finally, patient's exposure to unnecessary invasive diagnostic test such as biopsy could also be minimized.

Furthermore, TIME classification also provides a novel approach to assess patient's risk classification and guide immunotherapy treatments. A complete characterization of TIME also would provide more biological insights. Recently, adoptive cell treatments, such as CAR T cell therapy, have become increasingly popular. Immunotherapy treatments have shown that specifically reprogramming leukocytes (such as T cells) to target CD19 allows a patient's immune system to detect and fight off cancer cells. As opposed to chemotherapy, immunotherapy side effects are usually manageable and do not impede on quality of life. The most severe side effect of CAR T cell therapy is cytokine release syndrome (or cytokine storm), where patients suffer from hypotension and respiratory failures caused by an elevated level of cytokines serum. Fortunately, cytokine release syndrome can be treated and even cured by Actemra (or Tocilizumab), a new US FDA-approved drug [22]. Currently, immunotherapy treatments are used mainly on blood cancers (e.g., childhood acute lymphoblastic leukemia, multiple myeloma, diffuse large B cell lymphoma, etc.). CAR T cells therapy remains an experimental treatment on solid tumors since the treatment is mostly dependent on NK-led T cells infiltration which usually vary based on the TIME. Enrichments found in NK-cell-associated genes indicate potential new immunotherapy targets. Adoptive cell transfer using NK cells provides a new strategy to overcome tumor infiltration. Additionally, in contrast to T cells, NK cells can be transferred to a patient if the donor possesses similar HLA. NK cells could be used in a similar fashion to $\mathrm{T}$ cells to reprogram or reboot the immune system [23].

In conclusion, our results suggest that germline genomes of patients can be used to assess prognosis or diagnostic in cancer. We believe that germline usage and application in cancer represent a paradigm shift and could lead to many new and noninvasive applications in clinics which would lead to a better monitoring of cancer patients.

Financial \& competing interests disclosures

The authors have no relevant affiliations or financial involvement with any organization or entity with a financial interest in or financial conflict with the subject matter or materials discussed in the manuscript. This includes employment, consultancies, honoraria, stock ownership or options, expert testimony, grants or patents received or pending, or royalties.

No writing assistance was utilized in the production of this manuscript.

\section{Open access}

This work is licensed under the Attribution-NonCommercial-NoDerivatives 4.0 Unported License. To view a copy of this license, visit http://creativecommons.org/licenses/by-nc-nd/4.0/

\section{References}

1 Maistro S, Teixeira N, Encinas G et al. Germline mutations in BRCA1 and BRCA2 in epithelial ovarian cancer patients in Brazil. BMC Cancer 16(1), 934 (2016).

2 Chan SH, Lim WK, Ishak NDB et al. Germline mutations in cancer predisposition genes are frequent in sporadic sarcomas. Sci. Rep. 7(1), 10660 (2017).

3 Liaw D, Marsh DJ, Li J et al. Germline mutations of the PTEN gene in Cowden disease, an inherited breast and thyroid cancer syndrome. Nat. Genet. 16(1), 64-67 (1997).

4 De Queiroz Rossanese LB, De Lima Marson FA, Ribero JD, Coy CSR, Bertuzzo CS. APC germline mutations in families with familial adenomatous polyposis. Oncol. Rep. 30(5), 2081-2088 (2013).

$5 \mathrm{Hu}$ C, Hart SN, Polley EC et al. Association between inherited germline mutations in cancer predisposition genes and risk of pancreatic cancer. JAMA 319(23), 2401-2409 (2018).

6 Wang E, Zaman N, Mcgee S, Milanese JS, Masoudi-Nejad A, O’Connor-McCourt M. Predictive genomics: a cancer hallmark network framework for predicting tumor clinical phenotypes using genome sequencing data. Semin. Cancer Biol. 30, 4-12 (2015).

7 Milanese JS, Tibiche C, Zaman N et al. eTumorMetastasis, a network-based algorithm predicts clinical outcomes using whole-exome sequencing data of cancer patients. bioRxiv 268680 (2018). (Epub ahead of print) 
8 Milanese JS, Tibiche C, Zou J et al. Germline genetic variants associated with leukocyte-genes predict tumor recurrence in breast cancer patients. bioRxiv 312355 (2018). (Epub ahead of print)

9 Zou J, Wang E. eTumorRisk, an algorithm predicts cancer risk based on co-mutated gene networks in an individual's germline genome. bioRxiv 393090 (2018). (Epub ahead of print)

10 Xue X, Li J, Zou J et al. Inherited defects in natural killer cells shape tumor immune microenvironment, clinical outcome and immunotherapy response. bioRxiv 471912 (2018). (Epub ahead of print)

11 Forbes SA, Beare D, Boutselakis H et al. COSMIC: somatic cancer genetics at high-resolution. Nucleic Acids Res. 45(D1), D777-D783 (2017).

12 Alexandrov LB, Nik-Zainal S, Wedge DC, Campbell PJ, Stratton MR. Deciphering signatures of mutational processes operative in human cancer. Cell Rep. 3(1), 246-259 (2013).

13 Alexandrov LB, Nik-Zainal S, Wedge DC et al. Signatures of mutational processes in human cancer. Nature 500(7463), 415-421 (2013).

14 Helleday T, Eshtad S, Nik-Zainal S. Mechanisms underlying mutational signatures in human cancers. Nat. Rev. Genet. 15(9), 585-598 (2014).

15 Alexandrov LB, Stratton MR. Mutational signatures: the patterns of somatic mutations hidden in cancer genomes. Curr. Opin. Genet. Dev. 24, 52-60 (2014).

16 Vanunu O, Magger O, Ruppin E, Shlomi T, Sharan R. Associating genes and protein complexes with disease via network propagation. PLOS Comput. Biol. 6(1), e1000641 (2010).

17 Hofree M, Shen JP, Carter H, Gross A, Ideker T. Network-based stratification of tumor mutations. Nat. Methods. 10(11), 1108-1115 (2013).

18 Li J, Lenferink AEG, Deng T et al. Identification of high-quality cancer prognostic markers and metastasis network modules. Nat. Commun. 1, 34 (2010).

19 Karczewski KJ, Francioli LC, Tiao G et al. Variation across 141,456 human exomes and genomes reveals the spectrum of loss-of-function intolerance across human protein-coding genes. bioRxiv 531210 (2019). doi: https://doi.org/10.1101/531210

20 Dowsett M, Cuzick J, Wale C et al. Prediction of risk of distant recurrence using the 21 -gene recurrence score in node-negative and node-positive postmenopausal patients with breast cancer treated with anastrozole or tamoxifen: a TransATAC study. J. Clin. Oncol. 28(11), 1829-1834 (2010).

21 Albain KS, Barlow WE, Shak S et al. Prognostic and predictive value of the 21-gene recurrence score assay in postmenopausal women with node-positive, oestrogen-receptor-positive breast cancer on chemotherapy: a retrospective analysis of a randomised trial. Lancet Oncol. 11(1), 55-65 (2010).

22 Le RQ, Li L, Yuan W et al. FDA approval summary: tocilizumab for treatment of chimeric antigen receptor T cell-induced severe or life-threatening cytokine release syndrome. Oncologist 23(8), 943-947 (2018).

23 Davis ZB, Felices M, Verneris MR, Miller JS. Natural killer cell adoptive transfer therapy: exploiting the first line of defense against cancer. Cancer J. 21(6), 486-491 (2015). 